\title{
¿Cuál es el problema con el cálculo de utilidad?
}

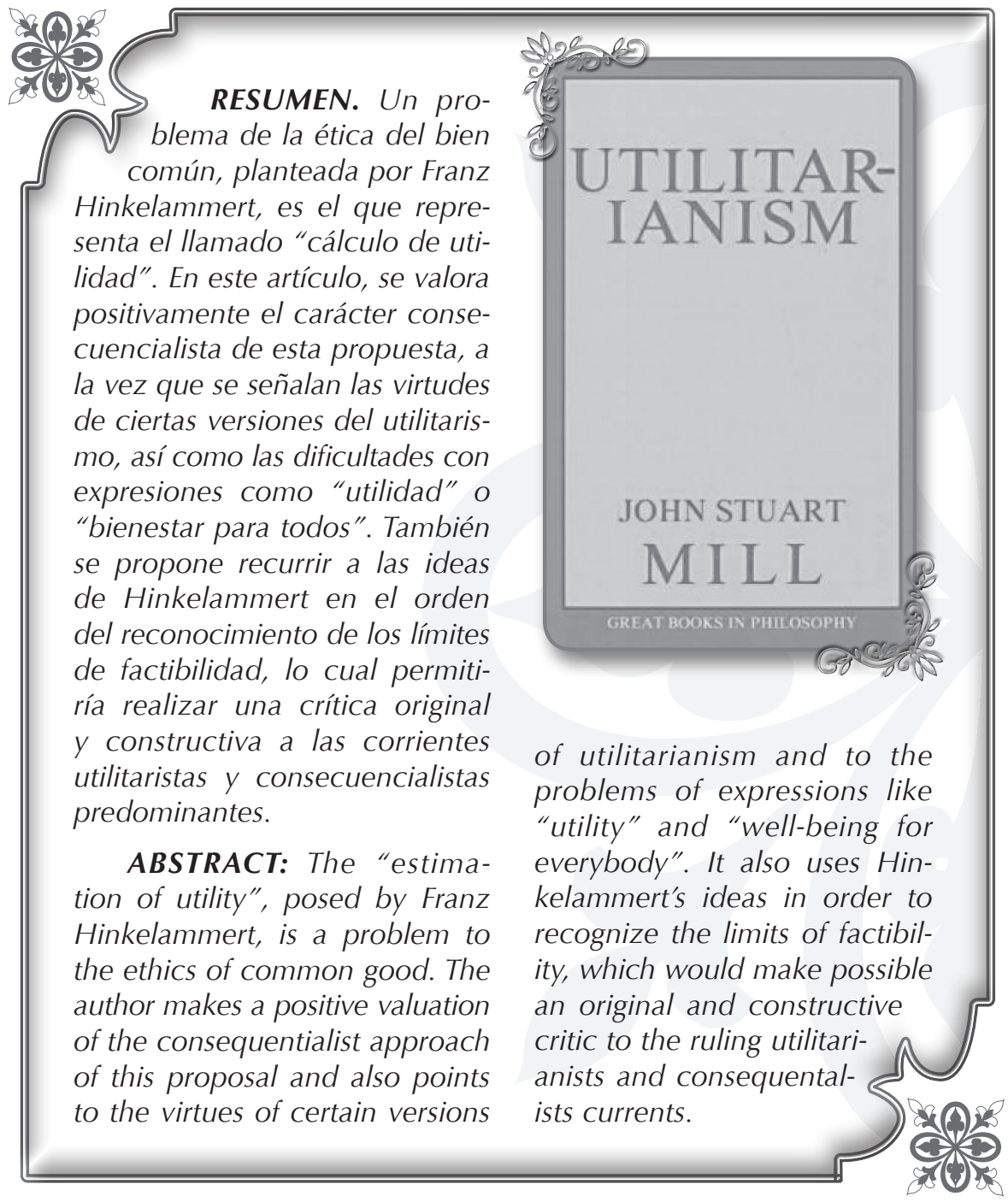



ás que la expulsión del "cálculo de utilidad", la ética del bien común que propone Franz Hinkelammert supone una reformulación de nuestras relaciones con él, debido a que es imposible erradicarlo del todo. Más bien, se trata de proponer una polaridad entre el cálculo de utilidad y la decisión en función del bienestar de todos. El cálculo es interpelado e "intervenido" por la racionalidad reproductiva, con lo que la perspectiva del agente es "relativizada", mediante su inserción en la perspectiva del sujeto. Así es como nos aparece el "bien común", que no es derivado de modo apriorístico, sino a partir del reconocimiento del sujeto viviente y de "lo que es útil para todos".

Esto último justifica una lectura consecuencialista de esta ética del bien común. Se trataría de un consecuencialismo que tomaría en cuenta esa polaridad entre el cálculo que se efectúa a nivel de la racionalidad medio-fin y el criterio que "obliga absolutamente", desde la perspectiva del sujeto. Pero no es sólo el consecuencialismo lo que se refuerza, sino que podríamos incluso pensar en una revalorización del utilitarismo. El mismo Hinkelammert señala la necesidad de la utilidad — en función del bienestar de todos y no sólo del beneficio de unos-, aunque se advierte sobre el problema de la imposibilidad de un cálculo exacto y total. El cálculo de utilidad tendría entonces que ser interpelado por el criterio de discernimiento, que se deriva del postulado trascendental de la razón práctica ("asesinato es suicidio") y que podríamos formular como el deber de reproducir la vida humana. Dice Hinkelammert:

"No se trata... de formular un cálculo de utilidad 'a largo plazo' o un cálculo de utilidad 'iluminado'. El cálculo de utilidad es el cálculo del individuo autónomo, que en su lógica produce precisamente aquellas fuerzas compulsivas de los hechos que desencadenan el proceso colectivo de autodestrucción, al cual se enfrenta la acción solidaria. Pero disolver este proceso destructor es útil (...)

La palabra 'utilidad' visiblemente expresa mediante un solo término dos cosas que se contradicen entre sí. La primera remite a lo útil necesario, la segunda al cálculo de utilidad (...)

En los dos casos, en el cálculo de utilidad [bienestar/beneficiol y en el caso de la utilidad beneficio/bienestar, que es útil para todos, y para todo, se trata del sujeto y de cada uno de los sujetos"1. 
Es claro que el problema no se encuentra en lo útil —la utilidad-, sino en la pretensión de cálculo exacto y total, así como en el carácter "atomista y fragmentario" de la acción —y la deliberación, cuando es el caso-, que realizan individuos limitados, pretendiendo realizar cálculos más allá de sus posibilidades. Esta es una ilusión trascendental, pero no significa que toda consideración de la utilidad e incluso que todo cálculo de la misma deba descartarse a priori. Al contrario, es el criterio de utilidad y su emplazamiento dentro de un enfoque en el que predominan las consecuencias el criterio para enfrentar la absolutización del cálculo, porque si no fueran nefastas las consecuencias para la humanidad, ¿qué otras razones podríamos tener para resistir a la lógica del (ilusorio) cálculo total?

Esto nos lleva a una primera idea que quiero plantear: La ética del bien común se clasificará dentro de las teorías consecuencialistas sobre lo que determina el acto correcto. ¿Qué es el consecuencialismo? "El consecuencialismo moral se define fundamentalmente como una teoría de lo correcto según la cual la corrección moral de nuestras acciones viene determinada por la bondad global de sus consecuencias" ${ }^{2}$. Esta es una consideración esencial para una teoría ética abierta, la cual se construye en oposición a las éticas que formulan valores abstractos y normas absolutas, así como a las éticas fun- cionales al mercado, que eluden la posibilidad de plantear valores "de por sí", necesarios para la vida, al mismo tiempo que justifican a las instituciones existentes, de manera tautológica o arbitraria. Frente a una ética meramente procedimental o frente a los enfoques liberales de los derechos humanos, que los derivan de una concepción restringida de la subjetividad, es necesario oponer un razonamiento que privilegie las consecuencias que tiene, para la vida humana, la aplicación de unas normas o la orientación en función de unos valores, por encima de la mera "observancia" de éstos o de aquéllas.

En este sentido, defender una postura consecuencialista significa estar de acuerdo con que el criterio para deliberar sobre la orientación de nuestras acciones es el fomento de determinados valores y fines, sin limitarnos únicamente a prescribir su "respeto". Asimismo, a la hora de evaluar nuestras opciones, el valor de éstas estaría determinado por el valor del pronóstico que podríamos hacer sobre los resultados de la acción correspondiente y las modificaciones que estaríamos introduciendo en el mundo, es decir, las consecuencias de esa acción ${ }^{3}$. Por ejemplo, la opción de garantizar la libertad de información en un país no puede evaluarse correctamente si no consideramos con cierta claridad que este valor debe ser fomentado y que deberá corresponder a unos "estados del mundo" preferibles (más libres y equitati- 
vos para todos). Pero se trata del fomento del valor y no su respeto incondicionado, por lo que estaría justificado prohibir la circulación de un periódico fascista o de una cadena de noticias que hace Ilamados a la violencia y al sabotaje, socavando la consolidación de una sociedad libre y equitativa.

¿Por qué es importante ubicar a la ética del bien común dentro de las teorías de corte consecuencialista? Hay al menos dos razones. Primero, porque de esa manera cobra fuerza el carácter a posteriori de sus fundamentos. No es una ética que formula normas fundadas en una esencia humana, un principio inquebrantable o fines determinados de antemano por un grupo de la sociedad. Es la evaluación de la acción mediante la evaluación de sus consecuencias para la vida de la humanidad lo que anima a la ética del bien común.

En segundo lugar, si queremos hacer de la ética del bien común una teoría ética, deberá posicionarse frente al abanico de teorías éticas existentes. Incluso si no pretendemos tal cosa, a lo mejor porque sólo nos dedicaremos a señalar los límites de los enfoques predominantes en ética, aún así deberíamos decir por qué no nos interesa señalar cómo debería procederse en la deducción de normas concretas y acciones que fomenten los valores, de tal manera que aquellos límites sean tomados en cuenta. $Y$ esto implica construir una teoría ética.
Pero hay algo más. Considero que la principal objeción al cálculo de utilidad, señalado desde la ética del bien común, coincide con lo que muchos han considerado como el argumento principal en contra del consecuencialismo: la presunción de que, en toda circunstancia, deberemos calcular nuestras elecciones. Es evidente que una gran cantidad de elecciones quedarían paralizadas por nuestras limitadas posibilidades de cálculo (como al intentar priorizar entre los bienes y servicios a los que deberían tener acceso los ciudadanos) o por la contradicción que implicaría el mero cálculo (como al pretender calcular las expresiones de amor que debería prodigar a mis hijos). Aún así, el consecuencialista podría argumentar que su enfoque no es sólo y únicamente una teoría de la deliberación sino una teoría de la justificación de las opciones. Es decir, si bien no siempre deberíamos proceder como calculadoras, al menos tenemos un buen criterio para justificar nuestras opciones ${ }^{4}$.

No espero que esta explicación resuelva todos los problemas que se plantean al consecuencialista estándar, pero sí quisiera que nos ayudara a pensar en su conexión con otro problema del consecuencialismo, frente al cual la ética del bien común tiene algo que aportar. Me refiero a lo que podemos llamar "situaciones límite". Yo diría que es la típica objeción al consecuencialismo: si nos guiamos por las consecuencias de nuestros actos, podríamos justificar las acciones más 
aborrecibles, como torturar a un terrorista para saber dónde se encuentra una bomba y salvar así muchas vidas, o como cuando decido quitar el respirador a mi tío anciano y moribundo, para conservar su herencia (que a él no le serviría de nada). Puede que sea demasiado suponer que una teoría ética, cuya finalidad es servirnos de guía en nuestra vida cotidiana, deba descartarse debido a situaciones en las que no es muy probable que nos encontremos. No obstante, la ética del bien común ha surgido precisamente de un análisis crítico de unas situaciones límite que son utilizadas para justificar políticas muy determinadas, cuya influencia en el rumbo que toma el mundo no es despreciable. Pensemos por ejemplo en los argumentos de Bush para justificar su "guerra al terrorismo".

Hay que señalar que este problema de las situaciones límite corresponde al uso peyorativo de la frase "el fin justifica los medios", un acostumbrado ataque a los consecuencialistas. Por su parte, Hinkelammert nos advertía que la superación del cálculo de utilidad no se resuelve mediante la inclusión de consideraciones sobre el "largo plazo", ya que el problema lo encontramos en el "cálculo del individuo autónomo"; por lo tanto, no hay que buscar en esa lógica de argumentación la salida al problema que plantea la expresión "maquiavélica". Pero esto no significa que la reducción del problema de lo útil a los meros resultados sea un problema extraño para los consecuencialistas. Como señala Amartya Sen, refiriéndose a las políticas que buscan garantizar el bienestar en una sociedad: "Existe una distinción entre los 'resultados finales' (es decir, sin tener en cuenta el proceso por el que se consiguen, incluido el ejercicio de la libertad) y los 'resultados globales' (teniendo en cuenta los procesos a través de los cuales se han conseguido los resultados finales)" $)^{\prime \prime}$. Deberíamos defender los resultados globales, ya que nuestras consideraciones sobre lo bueno o lo útil no sólo toman en cuenta el estado al que llegamos sino la manera como los alcanzamos. Si pudiéramos encontrar la tecnología necesaria para conducir a un grupo de personas a la plena satisfacción de sus necesidades básicas (resultado final), pero bajo un régimen en el que no hay lugar para la libertad y a costa de un número elevado de muertes, rechazaremos esa posibilidad, y preferiremos un régimen y tecnología que no sólo busque la satisfacción de las necesidades básicas de las personas sino el que éstas puedan alcanzarse en libertad y sin un derramamiento de sangre (resultado global).

¿Significa lo anterior que la ética del bien común es como cualquier consecuencialismo estándar? No lo creo. Podríamos sentirnos tentados de preguntar acerca de la razón por la que un consecuencialista como Amartya Sen introduce esta "distinción". ¿Será que subyace algún tipo de reconocimiento de 
"límites" de la condición humana, los cuales no pueden traspasarse? Cualquier consecuencialista $-y$ no sólo Sen- rechazaría cualquier alusión a "valores absolutos", es decir, independientes de los resultados y los pronósticos que enlazan estos con nuestras opciones. ¿Será que estamos ante el "retorno de lo reprimido", es decir, de los principios éticos apriorísticos, deontológicos? ¿O estaremos ante la irrupción de un límite para nuestras humanas posibilidades, que se nos aparece en la forma de un valor reconocido a posteriori?

Acá resulta esclarecedora la distinción entre valores absolutos y valores "de por sí", que ha formulado Hinkelammert. Estos últimos no se obtienen mediante derivaciones apriorísticas. Más bien, lo que sucede es que su negación no sólo provoca efectos perniciosos sino incluso la disolución del marco general de toda valoración. Al ser valores que se integran dentro de la elección entre la vida y la muerte, los valores de por sí son consideraciones acerca de las condiciones de posibilidad de la experiencia. Si no son afirmados, generan consecuen- cias que terminan por diluir la realidad, y con ello cualquier criterio de valoración. Como vemos, no se trata de una "retorno a la deontología", sino de la constatación de los límites para la misma acción racional, incluso si está dirigida por la búsqueda de la utilidad.

Pero, insisto, la ética del bien común no es sin más consecuencialista. Siendo su punto de partida el reconocimiento de una trascendentalidad al interior de la vida humana - la inclusión de las consideraciones acerca de los límites de la condición humana, las imaginaciones trascendentales, y la postulación de la subjetividad como realidad y como totalidad- proporciona elementos interesantes para mostrar ciertas insuficiencias del consecuencialismo. De especial interés es su presentación del problema en torno a los límites de imposibilidad, elementos ineludibles en la constitución de un pensamiento que tenga pretensiones de universalidad, racionalmente fundada. En este sentido, son interesantes algunas observaciones de los mismos consecuencialistas:

"[Según Shelly Kagan",] si tuviéramos un conocimiento cierto del destino de todos los seres, de la cantidad de bien que está en juego, tendríamos que estar motivados a actuar en pro del mayor bien global. Pero la ausencia de un conocimiento de tal viveza constituye, según Kagan, un defecto cognitivo para nosotros. $Y$ al reconocerlo como un defecto, Kagan no ve por qué no podemos estar motivados ahora a actuar como si tuviésemos ese tipo de conocimiento"7. 
Es curioso el parecido con el punto de partida de Hinkelammert, aun cuando la "solución" que se plantea se aleja de él notablemente. Kagan parte de una constatación muy similar a la que nos propone aquél —los límites para la condición humana-, pero los limita a "lo cognitivo". Desde la ética del bien común, diríamos que hay que llegar más lejos: se trata de límites para la acción, que provienen de nuestra condición de vivientes. El que tengamos deficiencias cognitivas es resultado, no principio. Además, la referencia a un tipo de razonamiento que emplea el "como si" - "no ve por qué no podemos estar motivados ahora a actuar como si tuviésemos ese tipo de conocimiento" ${ }^{\prime \prime}$ - expresa precisamente la lógica de la ilusión trascendental, que confía en que los límites no son más que una deficiencia cognitiva, superable con el paso del tiempo. Por el contrario, para Hinkelammert, estos límites son reconocidos racionalmente, mediante la postulación de la realidad como subjetividad trascendental. Son límites de imposibilidad, que surgen debido a la condición humana.
La falacia consiste en actuar como si poseyéramos un conocimiento perfecto o como si pudiéramos alcanzarlo en algún momento de la historia, pues esto genera acciones sociales, normas y valores que, eventualmente, aplastan a sujetos concretos. Y los resultados globales terminan siendo catastróficos. Para Hinkelammert, lo que no debemos suponer es que tengamos conocimiento perfecto. $Y$ esto es así porque la postulación subjetiva de la realidad se nos hace presente en su negación: asesinato es suicidio. Los sacrificios humanos por venir y la inminencia de la catástrofe nos obligan a invertir el razonamiento de Kagan: dado que no podemos tener conocimiento perfecto, entonces no debemos suponer que lo tenemos. Si bien es cierto que, para ciertos cálculos de utilidad, será metodológicamente imprescindible la suposición del conocimiento perfecto, éste no debe convertirse en la justificación de un mecanismo de totalización de la lógica medio-fin, que funcione como principio prescriptivo.

Desde una fuente opuesta a las observaciones de Kagan, las críticas de James Griffin al consecuencialismo vuelven sobre el asunto:

"[Según James Griffin,] si todo 'deber implica poder' no habrá normas que se sitúen fuera de las fronteras establecidas por las capacidades humanas. ${ }^{9}$ Las normas morales han sido configuradas por agentes limitados física y psicológicamente. Contamos con normas que se ajustan a nuestras capacidades, en las que depositamos una gran confianza, y que en gran parte no podemos aceptarlas y abandonarlas a voluntad"10. 
Hinkelammert también ha señalado, en diversas ocasiones, la idea medieval de que "lo que no se puede no se debe". Pero las alusiones de Griffin al problema de la factibilidad sitúan mejor el problema. Lo que muestra su argumentación acerca de la existencia de los límites de nuestra acción es que ésta no se reduce única ni fundamentalmente a las acciones de individuos aislados; nos las arreglamos estableciendo "normas", que se adaptan a nuestras capacidades, y que "no podemos aceptarlas y abandonarlas a voluntad". No sólo somos sujetos limitados, sino que nuestra acción contempla un elemento subjetivo fundamental: la acción se realiza sobre la base de las convenciones e igual sucede con las prescripciones.

La acción humana está lejos de poder ser explicada sobre la base de la mera acción individual. Por eso creemos que una revisión crítica de los planteamientos consecuencialistas debe introducir el tema de las instituciones, relacionándolo con el de esas capacidades limitadas y las normas que se "ajustan" a éstas. El reconocimiento de la misma conditio humana, de los límites de los sujetos en el orden de la factibilidad, no sólo exige repensar los supuestos antropológicos individualistas presentes en el consecuencialismo, sino, como señalábamos en alusión a Kagan, debe tratar de insertar en su argumentación el problema de la trascendentalidad del sujeto, que aparece en el reconocimiento de los límites de imposibilidad y de la manera como están presentes en la misma estructura de la razón. Ambas cuestiones están interrelacionadas. De otra manera, terminaríamos oponiendo al consecuencialismo puras razones "moralistas", apelando al egoísmo de sus planteamientos. Pero tal estrategia no sólo es demasiado estrecha sino, además, poco eficaz, ya que será sencillo mostrar que el problema de la motivación egoísta es algo secundario, si lo que nos interesa son los resultados, incluso si son globales. Más importante es señalar que las inconsistencias que surgen del no reconocimiento de los límites de factibilidad socavan la lógica misma de la argumentación consecuencialista.

Ahora bien, retomando nuestras reflexiones iniciales, ies posible que, desde la ética del bien común, pensemos en una recuperación del utilitarismo? Las coincidencias con el consecuencialismo las encontrábamos, entre otras cosas, porque en éste no hay necesariamente una absolutización del cálculo de utilidad y de los resultados finales, sino que se construye una ética abierta a la inclusión de toda clase de estrategias posibles, desde la perspectiva de que las acciones deben evaluarse en función de sus resultados globales. Es más, la concepción consecuencialista estándar - producir, siempre, el mayor bienestar global—, podría "Ilenarse" con un criterio utilitarista: el bien lo reconocemos en la utilidad que proporciona al mayor número, en 
el largo plazo, mientras que la utilidad coincide con la suma de los intereses individuales. No creo que esto presente dificultades insalvables, dado que, lejos de convertir a la utilidad en anatema, la ética del bien común la recupera. Pero esta recuperación se hace estableciendo un matiz fundamental: "La palabra 'utilidad' visiblemente expresa mediante un solo término dos cosas que se contradicen entre sí. La primera remite a lo útil necesario, la segunda al cálculo de utilidad". Esto útil necesario es la utilidad vista dentro de la perspectiva del sujeto y del bien común, lo cual transforma también el cálculo de utilidad, relativizándolo.
Útil necesario significa utilidad para todos. Pero también es utilidad para todo. Esto quiere decir que lo útil "para todos" se verifica, empíricamente, como consecuencias benéficas para individuos y grupos, $y$, trascendentalmente, como el bienestar para el conjunto de la humanidad. Y este "útil trascendental" se convierte en condición de posibilidad de toda posible evaluación de las consecuencias, aunque nunca puede ser realizado. Pero el paso del útil individual hacia el útil del cada uno universal es un paso necesario. El cálculo fragmentario no es anulado y tampoco "la perspectiva" del individuo, pero sí son integrados dentro de la consideración subjetiva del bienestar de todos:

"El cálculo de utilidad y la utilidad para todos, que se sobrepone a este cálculo de utilidad (utilidad para todos que incluye a la propia Naturaleza) no se pueden sustituir uno al otro. Si me dejo llevar por las coordenadas de mis intereses directos, según un principio de la inercia calculada, caigo en el cálculo de utilidad, del cual se originan las fuerzas compulsivas de los hechos. Sin embargo, no me puedo comportar siquiera fuera de esta relación con mis coordenadas de intereses directos. Estos se imponen a mi actuación. Por eso tengo siempre un punto de vista egocéntrico, lo que no significa necesariamente un punto de vista egoísta. Yo juzgo a partir de mí, con lo cual mis intereses calculados se me imponen. No obstante, en el mismo acto descubro (puedo descubrir) que mis intereses calculados se tornan en contra de mí mismo. Este descubrimiento implica a la vez el descubrimiento de que soy el otro y el otro soy yo"11.

Lo anterior presenta aún un problema: lo "útil para todos" no puede ser alcanzado por un cálculo, pero tampoco es garantizado por ninguna acción que pueda alcanzarlo. Siempre aparece como un límite. Eso 
es lo que significa que siempre se impongan los intereses calculados, no por un defecto en "nuestra moral" —que seamos egoístas, malos, etc.- sino porque la opción que se orienta en base a pronósticos es siempre fragmentaria. Pero debemos decidir si nuestra relación con los valores funcionará en base a nuestra capacidad para fomentarlos y para pronosticar los escenarios preferibles... o renunciar a una ética que toma en consideración la acción y sus resultados a posteriori. Aquí es donde vuelve a aparecer la perspectiva subjetiva en forma de postulado de la razón práctica: asesinato es suicidio. Y esto es así porque

"El ser humano, que se pone en el centro, tiene que descubrir en este mismo acto que él es el otro, y que, por tanto, él es el mundo. Si destruye al mundo, se destruye a sí mismo. Pero otra vez se trataría de poner al cálculo de utilidad en una posición subordinada, aunque esté siempre presente en el punto de partida, como también está en el punto de partida la actitud hacia el beneficio/bienestar de todos.

Nuestra sociedad de hoy, en cambio, transforma el cálculo de utilidad en un principio metafísico. Juzgado bajo este principio, lo egocéntrico parece ser lo natural, la solidaridad lo artificial; lo egocéntrico lo original, la solidaridad lo derivado. Así, un niño es juzgado como un ser egocéntrico original, un participante ideal del mercado, distorsionado por el aprendizaje posterior de la solidaridad. En realidad, el niño aprende la distinción de lo útil entre el cálculo de utilidad y la utilidad solidaria de todos, mientras parte de la unidad y conflictividad de ambos"12.

Cada uno de nosotros aprende a distinguir entre el cálculo egocéntrico y la utilidad necesaria, pero partiendo de "la unidad y conflictividad entre ambos", en vistas de que, si no se conservan las relaciones sociales mediante la suspensión solidaria del cálculo, todo lo útil se destruye. Esta "acción solidaria" es libertad. El sujeto libre es la libertad que deriva de la construcción del sujeto: "libertad frente al cálculo de utilidad"13. Pero también es importante señalar que lo útil se destruirá de igual manera si el cálculo desaparece totalmente. $\mathrm{O}$, más bien, si intentamos que desaparezca, ya que no puede desaparecer totalmente sino sólo subordinarse a la acción solidaria. Esto significa que, 
"en nombre de la eficiencia reproductiva hay que establecer límites, que no pueden ser calculables o el resultado de un cálculo (...) Ahora bien, los límites de este tipo son valores que garantizan la eficiencia reproductiva al limitar el espacio en el que la decisión puede ser tomada de modo legítimo, sobre la base de cálculos fragmentarios. Pero estos valores no pueden provenir de ningún cálculo. Se derivan del reconocimiento mutuo entre seres humanos, que incluye un reconocimiento de la vida de la propia naturaleza"14.

Volvemos a la necesidad de los "valores de por sí". Como límite del cálculo, establecen límites trascendentales a la misma valoración acerca del bienestar global. Pero no son valores absolutos, apriorísticos, sino que se encuentran en la constatación de los límites trascendentales de nuestra experiencia —que incluye al cálculo de utilidad-y en el reconocimiento mutuo entre sujetos. Además, son valores necesarios para garantizar otros valores, los cuales varían según los diversos modos de vida que se quiera construir.

No obstante, estos "otros valores" son también, por su parte, exigencias, por lo que pueden convertirse en sendos "límites" que, si se absolutizan, destruyen los valores necesarios, que en tanto son condición de posibilidad de la vida humana, lo son también de estos otros valores preferidos y con tendencia a ser absolutizados. $\mathrm{Si}$ bien los valores "constituyen el conjunto de preferencias sociales que pueden ser generalizadas más allá de la satisfacción inmediata de una necesidad"15, justo esta posibilidad de generalización los convierte en potenciales mecanismos de totalización de esas preferencias. Incluso el "valor de la vida" —no como valor "de por sí" derivado de los límites para nuestra experiencia, sino como valor absoluto- se vierte en mecanismos que aplastan la misma vida del sujeto ${ }^{16}$. Por ello, no sólo es importante discernir los valores desde el criterio subjetivo propuesto, sino que es preciso construir también propuestas de valores alternativos, con los cuales enfrentar los mecanismos institucionales que absolutizan preferencias y atentan contra la vida de la humanidad.

En este sentido, pienso que la mejor concepción ética sobre lo correcto es aquella que evalúa las opciones en función de los resultados y de los pronósticos correspondientes, y que esta teoría consecuencialista es la más adecuada para discernir los valores. Asimismo, creo que este discerni- 
miento debería replantear "el asunto del cálculo de utilidad" y responder con seriedad a las preguntas incómodas: “¿Por qué hemos de exigir alguna vez gestos que carecen de toda utilidad para alguien?"17. Si nuestro concepto central es el "bien común", ¿cómo comprender a éste de otra manera que no sea como un bien para alguien, es decir, útil? Puede ser que haya que volver a las teorías utilitaristas con una mirada abierta y renovada.

He intentado mostrar que, si pretendemos convertir a la ética del bien común en una teoría ética convincente, debemos incluir una reflexión acerca del lugar que ocupa al lado de otras teorías éticas. Y esto es importante, incluso si quisiéramos considerarla como una teoría ética "alternativa", no clasificable dentro de los parámetros de las teorías vigentes. Es sencillamente imposible eludir esta responsabilidad.

Notas

1 Hinkelammer, Franz y Mora, Henry; Coordinación social del trabajo, mercado y reproducción de la vida bumana, San José, DEI, 2001, pp. 324-325. Las cursivas y lo que se encuentra entre corchetes es mío.

2 Carrasco Barranco, Matilde; Conseuendas agenda y moralidad, Granada, Editorial COMARES, 2002, p. 1. Cursivas mías.

3 Cfr. Pettit, Philip; "El consecuencialismo", en Singer, Peter (ed.); Compendio de ética, Madrid, Alianza, 1995, p. 324-326.
Pero, además, me interesa destacar que hay razones "internas" que nos mueven a este ejercicio de posicionamiento en una dirección muy precisa, acercándonos a las posturas éticas consecuencialistas, entre las cuales ocupa un lugar destacado el utilitarismo (en sus muchas versiones). La primera razón es que la ética del bien común parte de una crítica al cálculo de utilidad, al mismo tiempo que recupera de éste aspectos fundamentales. La segunda, es que el núcleo fundamental de esta crítica -la ilusión trascendental del cálculo exacto, que no considera los límites de la condición humana- ofrece a los consecuencialistas elementos muy interesantes para sus propias discusiones acerca de la plausibilidad de una teoría que, no obstante sus limitaciones, posee muchos rasgos atractivos, entre los que podemos destacar su sencillez y apertura.

$4 \quad$ Cfr. Ibid.., pp. 328ss.

5 Sen, Amartya; Desamollo y libatad, Barcelona, Editorial Planeta, 2000, p. 45.

$6 \quad$ Cfr. Kagan, Shelly; "Donagan on the Sins of Consequentialism", Canadian Journal of Philosophy, 17 (1987) c. 8 y en Kamm, F.M.; "Non-consequentialism, the Person as an End-in-Itself and the Significance of Status", Philosophy and Public Affairs, 21 (1992), p. 359-362.

7 Carrasco Barranco, Matilde; Consecuencias, agencia y moralidad, op. cit., p. 137.

8 Las cursivas son mías. 
Hinkelammert, Franz; Cultura de Good and the Ambitions of Consequentialism", Social Philosophy and Policy, 9] (1992), p. 131, [y, también del mismo autor, en Value Judgement. Improving our Ethical Beliefs, Oxford, Clarendon Press,] 1996, p. 99 , c. VII.

10 Carrasco Barranco, Matilde; Consecuencias, agencia y moralidad, op. cit., p. 167.

11 Hinkelammert, Franz; Coordinación social del trabajo, mercado y reproducción de la vida bumana, op. cit., p. 326. Cursivas mías.

12 Ibid.., p. 327.

13 Cfr. Hinkelammert, Franz; El asalto al Poder Mundial y la violencia sagrada del Imperio, San José, DEI, 2003, p. 264ss.

la esperanza y sociedad sin exclusión, San José, DEI, 1995, p. 219. Cursivas mías.

15 Herrera Flores, Joaquín; "Hacia una visión compleja de los derechos humanos", en Herrera Flores, Joaquín (Ed.); El vuelo de Anteo. Derechos bumanos y crítica de la razón liberal, Bilbao, Desclée de Brouwer, 2000, p. 63.

16 Pensemos, por ejemplo, en los grupos "Pro Vida" que impulsan políticas y legislación para prohibir el aborto, ocasionando innumerables muertes entre las mujeres de nuestros países.

17 Goodin, Robert E.; "La utilidad y el bien”, en Singer, Peter (ed.); Compendio de ética, op. cit., p. 338. Cursivas mías. 